\title{
Research on the Decorative Design of Inorganic Composite Boards in Assembled Residential Houses
}

\author{
Yue Zhong ${ }^{1}$, Xiuyun $\mathrm{Li}^{1}$, Xun'e Zhou ${ }^{2}$ \\ ${ }^{1}$ Guangzhou Nanyang Polytechnic, \\ ${ }^{2}$ Guangdong Meisui Industrial Development CO., LTD. \\ Guangzhou Guangdong, China
}

\begin{abstract}
In the context of the industrialization of construction, prefabricated structures and interior decoration are developing rapidly. The residential integrated ceiling module selects one of the inorganic composite panels, inorganic high-strength sound absorbing board, as the main material, and introduces the light-weight, high-strength, energy-saving and environmentally friendly characteristics of inorganic highstrength sound-absorbing panels, as well as the acoustic structure and installation technology of the integrated ceiling in multiple spaces of the family residence, providing reference design scheme for the application of green technology in the residential integrated ceiling.
\end{abstract}

\section{Characteristics of inorganic high- strength sound absorbing board}

The inorganic high-strength sound absorbing board is a kind of green building material which is made of pure natural gypsum as a base material and other inorganic materials. The inorganic high-strength sound absorbing board has a formaldehyde emission of less than 0.1 , which is $1 / 15$ of the Chinese national standard. The fracture load is as high as $800 \mathrm{~N}$ or more. There are no cracks on the surface of the four-hour fire resistance test. The fire resistance belongs to A1 grade, and the antibacterial rate reaches $99 \%$. Compared with wooden sound absorbing boards, fabric sound absorbing boards, polyester fiber sound absorbing board and mineral wool sound absorbing board, inorganic high-strength sound absorbing board has high water absorption, which can be used in a relative humidity of $95 \%$. It has anti-mildew, moisture-proof and anti-insect properties and its deformation degree is less than $2 \mathrm{~mm}$. It doesn't ageing for a long time and remain well-defined shape for ten years." Porous structure" makes it have the function of "spontaneous breathing", which can adjust the indoor temperature and humidity balance. Installation is very convenient. The nail holding force of $34 \mathrm{~N} / \mathrm{mm}$ makes the boards fixing force strong. The same position can also be nailed repeatedly, and the nail holding effect remains unchanged.

Inorganic high-strength sound absorbing board has very good sound absorption properties. All building materials have certain sound absorbing characteristics. In engineering, materials and structures with high sound absorption coefficient (generally greater than 0.2) are called sound absorption materials or sound absorption structures $^{[1]}$. According to the structure of the material, the sound absorbing materials are generally mainly composed of three types: porous, resonance and special structure. Inorganic high-strength sound absorbing board is one of the most widely used porous sound-absorbing materials, and the sound absorption frequency ranges is 100-5000 $\mathrm{Hz}$, which can satisfy different medium and high frequency bands. Its structure is stable and easy to install. The boards has concave and convex modeling also has five good common surface coating decoration such as good waterproof, pearlescent, glaze, plastic spraying and UV texture. The inorganic high-strength sound absorbing board can avoid audio mixing in the process of sound reflection, thus improving the sound quality, making the sound absorption coefficient reach $0.75-0.80$, effectively reducing indoor noise.

With the development of technology, people's indoor acoustic space requirements for residential buildings are getting higher and higher. Inorganic high-strength sound absorbing board has become a popular high-quality building material that integrates features of sound purification, noise reduction, safety, comfort and environmental protection. Meanwhile, the good sound absorption effect makes it suitable for public, commercial, industrial and other civil buildings, especially star-rated hotels, hospitals, judicial units, scientific research institutions and other industries spaces that have special requirements for acoustics.

\section{Test of inorganic high-strength sound absorbing board}

The raw material for testing is a whole piece of inorganic high-strength sound absorbing board, which was assembled from 18 small boards. The inspection is based on GB/T20247-2006 "Acoustics-Measurement of sound 
absorption in a reverberation room" and GB/T167311997 "The graduation of sound absorption property for absorbent products". Sampling and sample delivery were carried out on April 11, 2018. The specification of the small plate was Y010 $(1200 \times 600 \times 15) \mathrm{mm}$, and China National Construction Engineering Quality Supervision and Inspection Center was commissioned to inspect the sound absorption coefficient and noise reduction coefficient of the plate. The sample number is SX1-20180049, and the test report shows that the noise reduction coefficient of the tested piece is $\mathrm{NRC}=0.50$, which meets the requirements of the class III index (Figure 1).

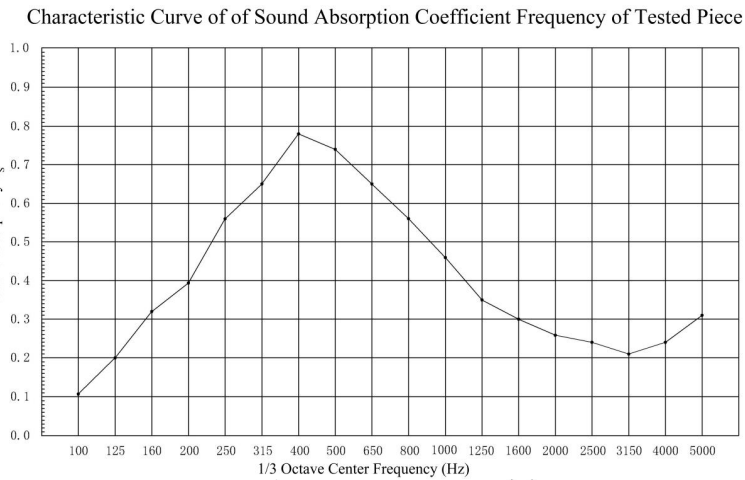

Figure1. Sound absorption curve of inorganic high-strength sound absorbing board

\section{Process and examples of inorganic high-strength sound absorbing board in residential integrated ceiling assembly modules}

\subsection{Design case for residential integrated ceiling}

In order to create a good acoustic residential space, three measures are adopted to solve noise pollution in the interior design of residential buildings: First, reduce the noise source: that is, to control the source of noise at the source of the noise, so as to reduce the noise intensity, like the transportation industry usually chooses low-noise equipment; Second, noise reduction on the sound propagation path: that is, setting obstacles in the sound propagation process to reduce the noise intensity, for example, the widespread use of sound insulation panels in various spaces to reduce noise; Third, implement noise protection: use ear protectors such as earplugs, earmuffs, or helmets to reduce noise intrusion. However, facts show that in daily life, people can't control all the sound sources, and it is difficult to control the noise source in time. In addition, people can not all wear noise reduction devices in frequent communication activities, which requires people to focus on the noise transmission path, in order to better achieve a quiet and comfortable home space.

In the current architectural interior design, the assembled integrated ceiling with inorganic high-strength sound absorbing board as the main material has good noise-reduction characteristics, which is generally recognized by the public. At the same time, the assembly module technology of the integrated ceiling has become an important part of the assembled interior technology of buildings. In the process of designing the residential ceiling module, compared with the traditional ceiling beam and column exposure, line collision and other outstanding problems, the integrated ceiling design can solve the defects well, meet the individual needs of users, effectively achieve diversified modeling and finely designed layout, which thus obtain a comfortable integrated roof-wall architectural aesthetic.

The first case is the selection of inorganic highstrength sound absorbing board for a residential integrated ceiling (Fig.2). In the design stage, the reserved openings and embedded parts are considered to have in light troughs, and the connection structure of the boards is integrated into the drawings, and factory prefabrication is adopted. It can better shorten the on-site opening and reduce the artificial opening error, also, control the gap of the hole to make the sound absorption effect of the residential ceiling better. In this case, the main material of the ceiling module has evenly aligned holes. For the integrated assembled ceiling of the residential space, the perforation rate of the inorganic high-strength sound absorbing board is not the same, and the general perforation rate is maintained at $2.29 \%-29.9 \%$. Different perforation rates are suitable for different functional home spaces, and combined with the frequency to select multistyle sound-absorbing panels, such as residential home theater space, in particular, it is necessary to pay attention to the spatial reflection, reverberation and reverberation of the space, and use the sound absorbing boards with different perforated rate to achieve the purpose of noise reduction.

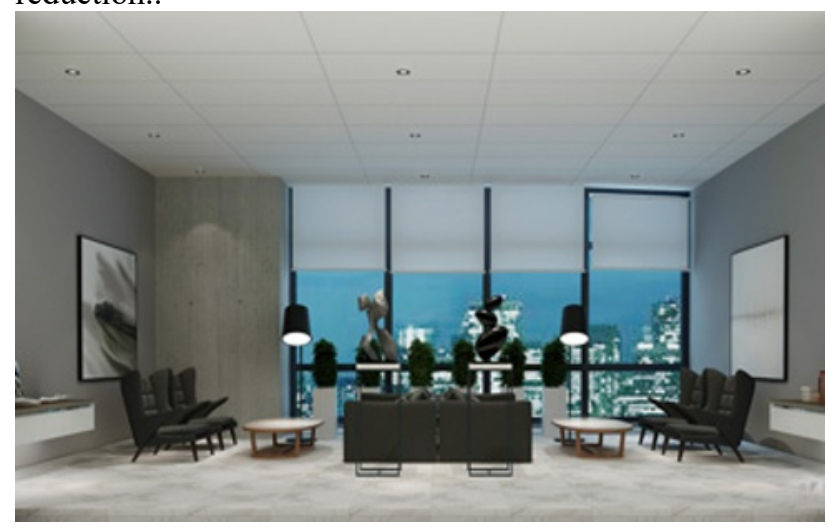

Figure2. Real view of integrated ceiling with inorganic highstrength sound absorbing board 


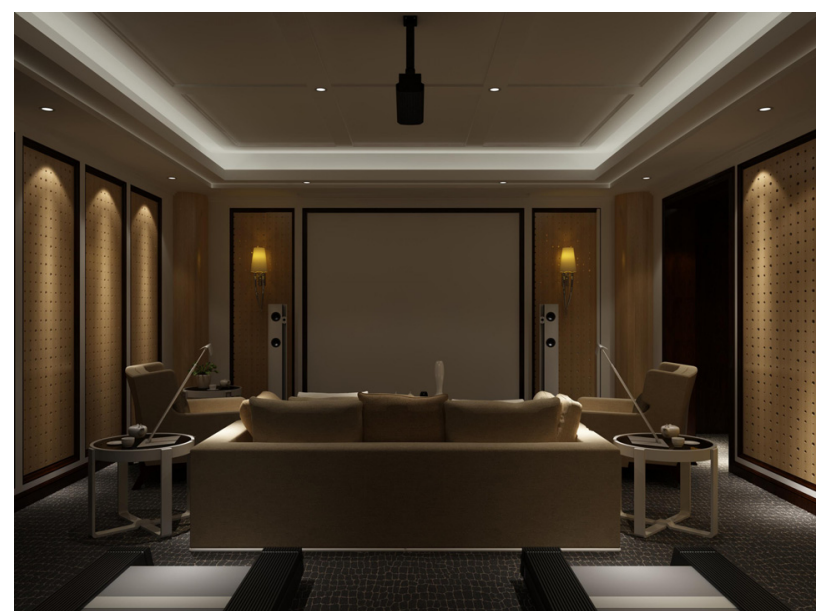

Figure3. The actual scene in the movie room of a residential family with inorganic high-strength sound absorbing board

\subsection{Assembly process}

The implementation of the assembled interior decoration system must connect the design technology and the construction process ${ }^{[3]}$. According to the principle of architectural acoustics, the sound-absorbing structure of the residential assembled ceiling module is made of an inorganic high-strength sound absorbing board with good sound absorption performance. The structure is fixed to the wall by the keel, and the cavity at the back of the board is filled with sound-absorbing rock wool to form a damping system, which makes the noise attenuate energy when it passes through the system, thereby forming an ideal sound-absorbing structure. The long-term accumulation of construction experience also proves that the sound-absorbing board filled with sound-absorbing cotton can effectively reduce noise ${ }^{[4]}$. In the case, the structure type of ceiling module is an inorganic highstrength sound absorbing board Y010 $+100 \mathrm{~mm}$ thick cavity, the surface density is $23 \mathrm{~kg} / \mathrm{m}^{2}$, the perforation rate of the board is $4.3 \%$ (Fig. 4-5), and the reverberation time is measured by interruption sound source method. The test results show that the noise reduction coefficient NRC of the ceiling module is 0.50 after installation.

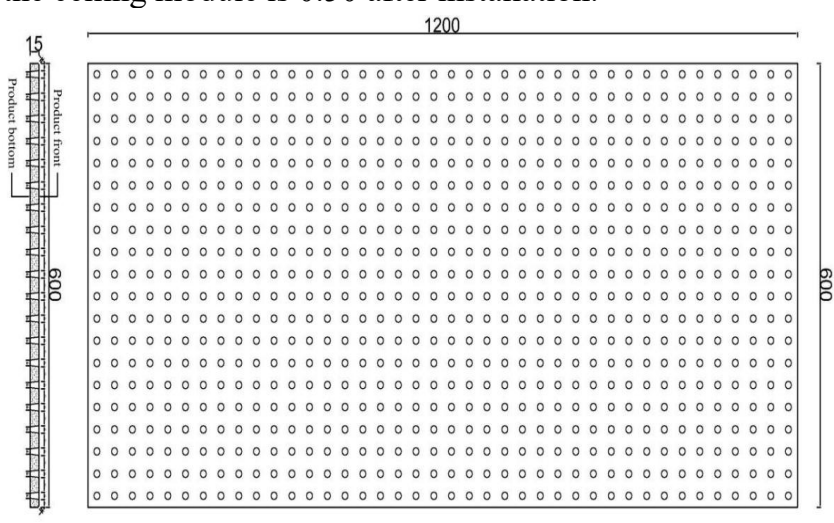

Figure4. Perforation plan of the tested piece Y010

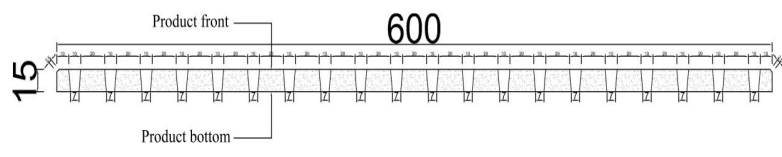

Figure5. Perforation size diagram of the tested piece

The ceiling is the main acoustic reflection surface, and the form of the ceiling should provide a useful early reflection sound ${ }^{[5]}$, while inorganic high-strength sound absorbing board is also suitable as a ceiling material. For the installation of the assembled ceiling module, considering that the high-frequency sound absorption of the living room is easy to absorb, the sound absorption of the surface of the ceiling module exposed to the air should be paid attention to in the design process. Sound absorption is subject to the limitation of the holes in the building decoration, the air outlet of the air conditioner and the lighting installation. The limitation of the hole should be reasonably designed in combination with the specific situation to avoid excessive gap absorption of low frequency sound. In order to meet the requirements of indoor beauty and tranquility, the upper space of ceiling module is used to absorb sound, the appropriate size of ceiling board holes is designed, and make the module holes open and close to adjust to sound absorption of the living room (Fig.6-7). What is worth mentioning is that properly increasing the fabric and sheet in the room is a necessary measure for noise control in home theaters. A variety of sound absorbing materials and sound absorbing structures can absorb low, medium and high frequency sounds respectively, thereby effectively reducing noise. In addition, proper positioning of the audiovisual equipment can achieve good audiovisual effects.

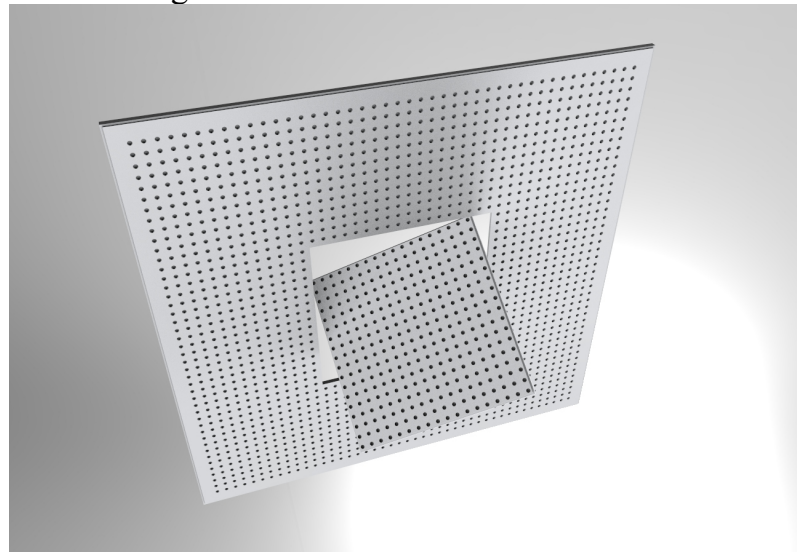

Figure6. Setting of the small section in the active hole of the ceiling module 


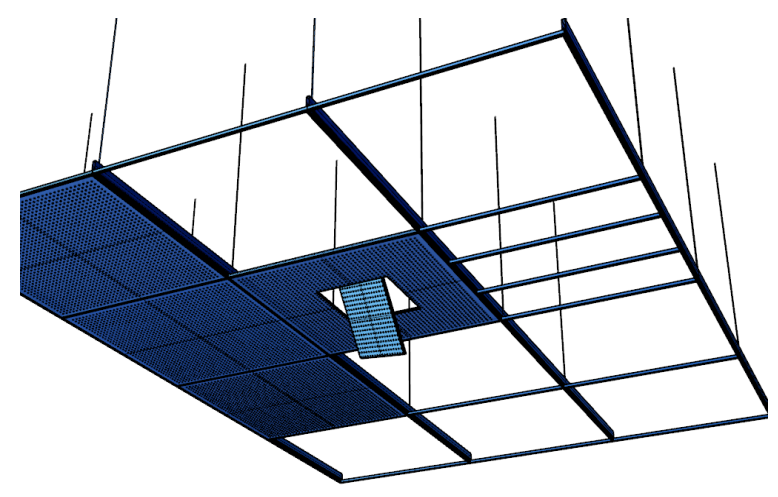

Figure7. Schematic diagram of the installation of the assembled integrated ceiling module

\subsection{Conclusion}

(1) The design of assembled ceiling module structure with perforation rate of $4.3 \%$ and surface density of $23 \mathrm{~kg} / \mathrm{m}^{2}$ inorganic high-strength sound absorbing board Y010 + $100 \mathrm{~mm}$ thick cavity can effectively make the residential space sound absorption and noise reduction, which meets the requirements of the contract;

(2) The installation of assembly ceiling module should consider the opening and closing of the module holes to adjust the sound absorption, so as to reduce the sound transmission and leakage phenomenon.

(3) The residential home theater space can utilize the characteristics of inorganic high-strength sound absorbing board, and a variety of sound insulation and sound absorption measures should be adopted in the ceiling module and the wall module to achieve good audio-visual effect of noise reduction.

\section{Prospect}

The assembly-type integrated ceiling has the advantages of optimizing and transforming the traditional ceiling decoration and construction mode, and is also an important part of the interior decoration of the fabricated architectural products ${ }^{[6]}$. Inorganic high-strength sound absorbing board has a very good sound-absorbing and noise-reducing characteristics, which can regulate the reverberation effect of the interior sound. It also can purify the sound quality and create a quiet living room environment and show that is a green new environmental protection technology building material. In the era of rapid development of technology innovation industry, it is expected that in the near future, inorganic high-strength sound absorbing board can achieve high-efficiency soundabsorbing performance of materials in different frequency bands through the research and development of composite processes, and be applied in various fields with the times.

\section{Acknowledgment}

This work was supported in part by the Program of major scientific research projects of Guangdong universities at provincial level Research on the Modular Design and Integrated Assembly of Green and Environmental Protection Housing Hanging Top (2017GkQNCX126)

\section{References}

1. Wu Shuoxian. Architectural Acoustic Design Principle [M]. China Construction Industry Press, 2000.

2. Ding Lei. Simulation and Test of Architectural Acoustics of Live Studio in Jiangyin Broadcasting Station[J]. Audio Engineering, 2017, 41(6), pp.1-11.

3. Peng Dianyong, Zhao Chunting, Liu Gang. Practice of Assembly Innerior Decration System[J]. City \& house, 2018 (1), pp.42-47.

4. Wu Chengge, Tian Hong, Wang Huaiyu. Study of the Foamed Aluminum and Absorbent Cotton Using in Acoustic Panel of Noise Barrier[J]. Journal of Xingtai Polytechnic College,2015(1), pp.87-90

5. Wang Hongwei, Yang Yajie, Zhang Long. Architecture Acoustic Design of Cantonese Opera Art Museum Theatre in Guangzhou[J]. Huazhong Architecture, 2017 (2), pp.32-36.

6. Li Xiuyun, Zhong Yue. Study on Process Technology of Integrated Ceiling Assembly Module with GRG Building Materials in Residential Buildings[J]. Guangdong Architecture Civil Engineering, 2018, 25 (05), pp. 63-65. 